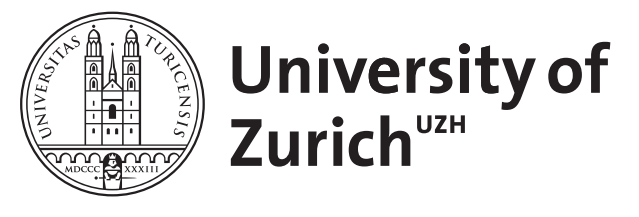

\title{
Cognitive dysfunctions in chronic cocaine users
}

\author{
Quednow, Boris B ; Vonmoos, Matthias
}

\begin{abstract}
After nearly three decades of research, there is still no clarity on the causal relationship between chronic cocaine use and cognitive functioning. However, the current literature consistently suggests that broad cognitive impairments occur in not only dependent cocaine users but also recreational cocaine users, with impairments involving attention, working memory declarative memory and executive functions. Moreover, it was recently shown that chronic cocaine users exhibit specific deficits in sociocognitive abilities, which very likely contribute to the social dysfunctions that occur in their daily lives. Correlation analyses and initial longitudinal studies indicate that most cognitive deficits are at least partially cocaine-induced. Accordingly, there is also some evidence regarding the recovery of cognitive functions in decreasing and long-term abstinent users.
\end{abstract}

DOI: https://doi.org/10.1016/B978-0-12-803750-8.00040-3

Posted at the Zurich Open Repository and Archive, University of Zurich ZORA URL: https://doi.org/10.5167/uzh-139590

Book Section

Originally published at:

Quednow, Boris B; Vonmoos, Matthias (2017). Cognitive dysfunctions in chronic cocaine users. In: Preedy, V R. The Neuroscience of Cocaine. London: Academic Press, 395-405.

DOI: https://doi.org/10.1016/B978-0-12-803750-8.00040-3 


\section{Cognitive dysfunctions in chronic cocaine users}

Matthias Vonmoos [1] and Boris B. Quednow

\section{[1] Corresponding author}

Matthias Vonmoos, PhD, MA

Experimental and Clinical Pharmacopsychology

Department of Psychiatry, Psychotherapy and Psychosomatics

Psychiatric Hospital of the University of Zurich

Lenggstrasse 31

CH-8032 Zurich, Switzerland

Tel.: 0041-44-384-2666

Fax: 0041-44-384-3396

E-Mail: matthias.vonmoos@bli.uzh.ch

\section{Additional author}

Boris B. Quednow, PhD

Experimental and Clinical Pharmacopsychology

Department of Psychiatry, Psychotherapy and Psychosomatics

Psychiatric Hospital of the University of Zurich

Lenggstrasse 31

CH-8032 Zurich, Switzerland

Tel.: 0041-44-384-2777

Fax: 0041-44-384-3396

E-Mail: quednow@bli.uzh.ch

\section{Department contact}

Michael D. Wunderli, MSc

Experimental and Clinical Pharmacopsychology

Department of Psychiatry, Psychotherapy and Psychosomatics

Psychiatric Hospital of the University of Zurich

Lenggstrasse 31

CH-8032 Zurich, Switzerland

Tel.: 0041-44-384-27601

Fax: 0041-44-384-3396

E-Mail: michael.wunderli@bli.uzh.ch

\section{Word count}

6600 words

0 tables

4 figures

65 references

1772 words in references

\section{Submitted}

June $1^{\text {st }}, 2016$

\section{Abbreviated title}

Cognitive dysfunctions in cocaine users 


\begin{abstract}
After nearly three decades of research, there is still no clarity on the causal relationship between chronic cocaine use and cognitive functioning. However, the current literature consistently suggests that broad cognitive impairments occur in not only dependent cocaine users but also recreational cocaine users, with impairments involving attention, working memory declarative memory and executive functions. Moreover, it was recently shown that chronic cocaine users exhibit specific deficits in socio-cognitive abilities, which very likely contribute to the social dysfunctions that occur in their daily lives. Correlation analyses and initial longitudinal studies indicate that most cognitive deficits are at least partially cocaine-induced. Accordingly, there is also some evidence regarding the recovery of cognitive functions in decreasing and long-term abstinent users.
\end{abstract}

\title{
Keywords
}

Neuropsychology, cognition, social cognition, stimulants, crack, impulsivity, decision-making, empathy, theory-of-mind, reversibility 


\section{List of abbreviations}

ADHD: Attention-deficit/hyperactivity disorder

DSM: Diagnostic and Statistical Manual of Mental Disorders

EMCDDA: European Monitoring Centre for Drugs and Drug Addiction

$\mathrm{ZuCo}^{2} \mathrm{St}$ : Zurich Cocaine Cognition Study 


\section{Introduction}

Since cocaine dependence first appeared as a diagnostic category with the publication of the DSM-III$\mathrm{R}$, research studies have been conducted to examine the relationship between repeated cocaine use and its physical, psychological and neurobiological consequences. In this context, a growing body of literature has consistently linked ongoing chronic cocaine use to different aspects of cognitive functioning. Although drug use is generally considered to be driven by positive and negative affective or emotional states (Koob, 2013), it also comprises a substantial volitional component based on cognitive processes guiding decision-making and behaviour (Garavan \& Hester, 2007). Hence, it is important to investigate cognitive changes in cocaine users so as to attain a better understanding of the mechanisms of recreational and controlled cocaine use and its transition to dependent use as well as successful cocaine abstinence and relapse (Garavan \& Hester, 2007; Gould, 2010). Moreover, as cognitive functioning seems to be relevant at each stage of the drug use cycle (Garavan \& Hester, 2007), drug addiction might generally be considered a disorder of altered cognition (Gould, 2010) - or more specifically, a form of pathological learning and memory (Hyman, 2005). Consequently, this raises the essential question regarding to what extent the impaired cognitive functions of cocaine users are pre-existent traits, drug-induced consequences or an interaction of both. The clarification of this issue is important with regard to not only the development of more complex addiction models but also the improvement of prevention, treatment and post-treatment strategies in cocaine addiction.

\section{Cognitive functioning in dependent cocaine users}

Since the 1980s, researchers have endeavoured to examine the impact of chronic cocaine use on cognition, primarily by focusing on chronic and dependent cocaine users. The first broad quantitative review, conducted by Jovanovsky et al. (2005), included 15 empirical studies published between 1987 and 2002, which incorporated cocaine users who met the DSM-III-R or DSM-IV criteria for cocaine abuse or dependence $(n=481)$ and matched control groups ( $n=586$ controls). Their results revealed that chronic cocaine use had strong effects on different measures of attention (Cohen's $d=0.40-1.10$ ), moderate to strong effects on visual memory and working memory $(d>0.50)$ and rather small effects on verbal fluency as well as other language and sensory-perceptual functions $(d<0.30)$. Moreover, research pertaining to the heterogeneous concept of executive functioning revealed mixed findings, primarily depending on task requirements and difficulty levels. The major limitation of this review was the wide variety of abstinence periods in the analysed studies (0-1076 days). However, the mean abstinence time in the vast majority of the included studies was clearly more than three days, a time period that nowadays is commonly estimated to be relevant for acute and post-acute (withdrawal) effects of cocaine (Potvin et al., 2014). Insofar, the main results of Jovanovsky et al. (2005) can be interpreted as non-acute cognitive effects of cocaine use. 
Eight years later, Spronk et al. (2013) updated the review by Jovanovsky et al. (2005) by analysing 14 studies on acute effects and 63 studies on long-term effects that were published between 2003 and 2012. This was the first review that systematically distinguished between acute (defined as following cocaine administration in a laboratory setting) and long-term effects. Notably, long-term effects were broadly defined as effects in individuals with a history of cocaine use compared with cocaine-naive controls; a minimal abstinence time was not considered. Therefore, nearly half of the included studies on long-term effects had a minimal abstinence duration that was either unknown or less than three to four days. In addition,, Spronk et al. (2013) included seven preliminary and - with one exception (Rahman \& Clarke, 2005) - relatively small studies on recreational cocaine users with sample sizes of $\mathrm{n} \leq 20$. Given that 56 of the 63 reviewed studies focused on cocaine users with a diagnosed abuse or dependence, the main results of this systematic review might be more valid for chronic and dependent users than for occasional or recreational users. Despite these limitations, Spronk et al. (2013) reported - in agreement with the previous review by Jovanovsky et al. (2005) - that chronic cocaine use is characterised by broad cognitive impairments in the domains of attention and memory and various sub-processes of executive functions (impairments in reward-based decision-making and response inhibition, mild impairments in cognitive flexibility, indications for deficits in performance monitoring).

A third systematic review, conducted by Potvin et al. (2014), considered the limitations of previous reviews by (a) including only studies with cocaine users who were diagnosed as abusers or dependents in accordance with $D S M$-criteria and (b) differentiating between short (positive urine screening), intermediate ( $\leq 12$ weeks) and long-term ( $\geq 20$ weeks) abstinence. This review included 1452 chronic cocaine users and 1411 controls from 46 studies (including 14 of 15 studies from the review by Jovanovski et al., 2005). In accordance with the two previously discussed reviews, the results of Potvin et al. (2014) suggest significant impairments across multiple cognitive domains for the intermediate abstinence duration. Again, attention $(d=0.59)$ seemed to be more impaired than working memory $(d=0.52)$ and some specific memory sub-domains (verbal/visual, learning/memory; $d=0.43-$ 0.56). Moreover, some smaller deficits were found in a not-further-diversified executive functions domain $(d=0.32)$. Furthermore, their results indicate that impairments were smaller after short-term abstinence than after intermediate abstinence, thus confirming some previous findings indicating that acute and residual cocaine effects influence cognitive functioning and mask some persisting neuropsychological decrements (Spronk et al., 2013).

Whereas studies performed in the 1990s produced some ambiguous results, the more recent literature on dependent cocaine users draws a widely consistent picture of broad and relatively unspecific cognitive impairments in cocaine users. The rare inconsistencies within the recent publications are probably based on theoretical and methodological issues such as small sample sizes and different abstinence durations, as well as different cognitive domain definitions (notably, the executive function domain is still ill-defined, encompassing a wide range of concepts and tasks), psychiatric co- 
morbidities and patterns/histories of cocaine or polydrug use. Moreover, as research has shown (Magura \& Kang, 1996; Vonmoos et al., 2013), a considerable percentage of alleged cocaine users are probably not telling the truth about their actual drug use pattern and history. The lack of an objective verification of self-reported drug intake in most studies remains a major weakness in this research field. However, within the framework of the large Zurich Cocaine Cognition Study $\left(\mathrm{ZuCo}^{2} \mathrm{St}\right)$, in which urine and hair toxicology analyses were performed to characterise objectively not only recent drug use but also drug use over the past six months, Vonmoos et al. (2013) confirmed the results of the current literature in a large cross-sectional analysis by describing broad cognitive impairments in 30 dependent cocaine users (Figure 1). Again, their results demonstrated that impairments were not specific; that is, they affected all investigated cognitive domains - attention, working memory, declarative memory and executive functions - with moderate to large effect sizes $(d=0.74-1.00)$

(Figure 2).

\section{Cognitive functioning in recreational/occasional cocaine users}

Whereas most studies have focused on the dependent use of cocaine, only limited research has systematically investigated the cognitive effects of only occasional or recreational drug intake on nondependent cocaine users. However, as this user group accounts for the majority of cocaine users (Anthony, 2002; EMCDDA, 2015), potential cognitive changes yield higher ecological validity and are, thus, especially interesting with regard to prevention efforts. Compared with research on dependent cocaine users, the analysis of this non-dependent user group has provided evidence of not only some considerable advantages but also some additional barriers. On the one hand, recreational cocaine users are - as data from our $\mathrm{ZuCo}^{2} \mathrm{St}$ have shown - generally less burdened by psychiatric comorbidities and polytoxic drug use (Quednow, 2016). Moreover, because recreational cocaine use can be considered an intermediate step towards addiction (Gould, 2010), the separate analysis of nondependent users allows a clear distinction between aspects specifically related to addiction and those presumably related to occasional use. On the other hand, there is currently no standard classification of recreational cocaine use. Whereas cocaine abuse and dependency are still diagnosed based on the DSM-IV criteria - or, recently, are based on substance use disorder symptoms in DSM-V - the current literature continues to provide different definitions of recreational cocaine use, with most definitions based on the recent consumption pattern. Additional barriers to the comparability of studies with recreational cocaine users (similar to those barriers described in the studies on dependent users) are the variations in the minimal abstinence periods (ranging from two days to two weeks) and the mostly missing objective toxicology measures. Although the different definitions might pose a problem with regard to the comparability of these studies, one should keep in mind that the majority of the studies on recreational cocaine use shared two important features: these studies usually required recreational users to have a certain minimal use of cocaine in the last month $(\geq 0.5 \mathrm{~g})$ and not explicitly meet the previously mentioned DSM-IV criteria for cocaine dependence. 
The limited literature available, consisting of studies with mostly small and sometimes overlapping samples of recreational cocaine users, indicates that already small and infrequent doses of cocaine affect different cognitive components. More specifically, the published studies described deficits in attention, visuospatial perception, components of verbal memory (Rahman \& Clarke, 2005), inhibitory behavioural control (Colzato et al., 2007), scope of visual attention (Colzato et al., 2009b), cognitive flexibility but not working memory (Colzato et al., 2009a), control of saccadic eye-movement (inhibition-of-return) (Colzato \& Hommel, 2009), sustained attention, attentional shifting, spatial memory (Soar et al., 2012), cognitive control in the sense of conflict management (Sellaro et al., 2014) and inhibitory attentional processes (Soar et al., 2015). Furthermore, recreational stimulant users with cocaine as the primary drug of use showed memory performance deficits in verbal learning, recall and recognition domains (Reske et al., 2010). Recently, Vonmoos et al. (2013) investigated the largest sample of recreational cocaine users $(n=68)$ to date; in addition, this sample was the only one thus far to undergo urine and six-month hair toxicology analyses as objective measures of cocaine use intensity. The results of this study were consistent with the previous literature suggesting considerable and broad and widely unspecific cognitive impairments even in recreational users. Vonmoos et al. (2013) reported that similar to dependent users, recreational users exhibited significant impairments in the domains of attention, working memory, memory and executive functions, all with moderate effect sizes $(d=0.39-0.44)$. However, in all four domains, recreational cocaine users performed intermediately between controls and dependent users (Figure 2).

\section{Relationship between the severity of cocaine use and cognitive functioning}

In summary, the aforementioned findings suggest that the cognitive performance differences between recreational and dependent users reflect either a qualitative difference between the two user groups (addicted vs. non-addicted) or a dimensional relationship between the severity of cocaine use and cognitive impairments. The latter hypothesis is supported by the fact that several studies have found negative correlations between cognitive performance and the severity of cocaine use, as, for example, measured by dose of cocaine (g/week) (Bolla et al., 2000; 1999), cumulative cocaine dose, duration of cocaine use and cocaine metabolites in hair (Vonmoos et al., 2013). These dose-response relationships have been further confirmed by an increased risk of cognitive impairment at increasing cumulative doses of cocaine (Vonmoos et al., 2013). Whereas a lifetime consumption of $>10 \mathrm{~g}$ was associated with a $~ 50 \%$ risk for mild cognitive impairment (below -1 standard deviations from the mean of the controls), this risk increased to approximately two thirds for those with a lifetime use $>500 \mathrm{~g}$ and, even further, to $75 \%$ for those with a cumulative lifetime dose of $>1 \mathrm{~kg}$ (Figure 3).

As investigated in a cross-sectional study design, these results suggest that cognitive impairments might be at least partially cocaine-induced (Vonmoos et al., 2013). These findings are in line with previous studies on chronic cocaine self-administration in rhesus monkeys, which also suggested that 
some alterations in cognitive functioning might be cocaine-induced (Gould et al., 2012; Porter et al., 2011).

\section{Cocaine-induced cognitive deficits are drug-induced and partially reversible}

Even though the cross-sectional literature initially suggested that deficits in cognitive functioning might be at least partially cocaine-induced, longitudinal studies are needed to support this hypothesis. Moreover, the reversibility of cognitive dysfunction can be investigated only with longitudinal study designs. As longitudinal studies are very costly and time-consuming, there are currently only a few studies that have investigated cognitive changes in cocaine users over longer time periods. To date, we are aware of five cross-sectional (Bolla et al., 1999; De Oliveira et al., 2009; Di Sclafani et al., 1998; Rosselli \& Ardila, 1996; Rosselli et al., 2001) and four longitudinal studies (Bauer, 1996; Bolla et al., 2000; Di Sclafani et al., 2002; van Gorp et al., 1999) investigating the effect of long-term abstinence ( $\geq 1$ month) on cognition in cocaine users. Most of the studies with short abstinence periods suggested stable and persistent cognitive impairments; however, the two studies with the longest abstinence durations - six months (Di Sclafani et al., 2002) and longer (De Oliveira et al., 2009) both suggested cognitive recovery for abstinent cocaine users with regard to working memory (Di Sclafani et al., 2002) and verbal declarative memory (De Oliveira et al., 2009). Whereas Di Sclafani et al. (2002) themselves critically noted that they could not confirm such improvements for any other domain and therefore speculated that these working memory improvements might be based on practice effects, De Oliveira et al. (2009) suggested that some of their presented recovery effects could indeed have been caused by abstinence, thus indicating the reversibility of cognitive deficits. Accordingly, the aforementioned review by Potvin et al. (2014) analysed five studies on cocaine users with abstinence periods $\geq 20$ weeks and indicated that cognitive impairments remain stable during the first few months of abstinence but probably dilute after some months.

The clearest limitations of the long-term abstinence studies cited here were (a) the general lack of toxicological verifications of cocaine use and abstinence periods (most studies performed no toxicology tests or only random urine screenings), (b) the sometimes very rudimentary information on the severity of previous drug use and (c) the relatively brief follow-up intervals. Furthermore, these studies focused exclusively on cocaine users who ceased their consumption, and until recently, there were no published analyses investigating the cognitive development in individuals who increased their cocaine consumption. Vonmoos et al. (2014) were the first to investigate the relationship between changes in the intensity of cocaine use and the development of cognitive functioning. Their sample was tested twice with a one-year interval between testing, and the intensity of cocaine use was objectively determined by quantitative six-month hair toxicology analyses. The analyses of hair cocaine concentrations revealed that one third of users $(n=19)$ displayed strongly increased cocaine levels (mean $+297 \%$, mean cocaine concentration $+30.4 \mathrm{ng} / \mathrm{mg}$, SD $61.9 \mathrm{ng} / \mathrm{mg}$ ), one third $(\mathrm{n}=19)$ showed substantially decreased concentrations $(-72 \%,-10.6 \mathrm{ng} / \mathrm{mg}$, SD $26.7 \mathrm{ng} / \mathrm{mg})$ and one third 
$(n=19)$ had not changed their consumption pattern. However, the stable user group included rather moderate users with much lower cocaine contamination in hair compared with the other two user groups (Vonmoos et al., 2014). In this study, substantially increased cocaine use within one year led to further cognitive performance deterioration, foremost in the working memory. By contrast, and as expected from the previously mentioned studies by Di Sclafani et al. (2002) and De Oliveira et al. (2009), decreased cocaine use generally improved cognition and showed the strongest enhancements in working memory and declarative memory. Moreover, of the 19 decreasing users, eight did not reach a commonly accepted hair cocaine concentration cut-off of $0.5 \mathrm{ng} / \mathrm{mg}$ at follow-up (Cooper et al., 2012), and, thus, they were considered to have been abstinent for at least three to six months. All these eight ceasing users seemed to recover completely and attained a cognitive performance level comparable to that of the controls (Figure 4). As this abstinent user group reported a cumulative cocaine lifetime dose of $700 \mathrm{~g}$ on average, it reached a lifetime dose amount that has been previously shown to increase the risk for cognitive impairment clearly (Vonmoos et al., 2013). However, it remains unclear whether there is a 'point of no return' beyond which a full recovery cannot be expected.

\section{Confounding and moderating factors}

Given that cocaine use is frequently associated with symptoms of attention-deficit hyperactivity disorder (ADHD) (Wilson, 2007) and depression (Swendsen \& Merikangas, 2000), it should be noted that both co-morbidities are also important modulators of cognitive functioning in cocaine users (Vonmoos et al., 2013). Recent research studies found that the combination of ADHD and cocaine consumption seemed to have a cumulative aggravation of detrimental effects on cognitive performance (Vonmoos et al., 2013; Wunderli et al., 2016). By contrast, only a weak additive impact of depressive symptoms on cognitive performance was found in recreational users, but not in dependent users (Vonmoos et al., 2013).

Another important modulator for cognitive functioning seems to be the age-of-onset of cocaine use. Individuals who started their cocaine consumption before the age of 18 years showed stronger cognitive impairments than users with a later onset - even when the data were adjusted for duration of use or age (Vonmoos et al., 2013). Moreover, the longitudinal analysis showed that early onset of use was associated with reduced recovery of working memory when the cocaine use was decreased (Vonmoos et al., 2014). In summary, these data suggest that early onset of cocaine use might be a risk factor for sustained cognitive impairment following chronic cocaine use.

\section{Social cognition}

Another important aspect of cognitive functioning that is quite relevant in different mental disorders (including cocaine addiction) is social cognition (Verdejo-Garcia, 2014). Given that social problems 
are well known in drug users and that socio-cognitive abilities have been demonstrated to play important roles in psychiatric disorders such as depression (Weightman et al., 2014) and schizophrenia (Couture et al., 2006), it was analogously proposed that social cognition and interaction may also play essential roles in the origin and course of drug addiction (Volkow et al., 2011). Until recently, systematic and experimental investigations broadly characterising and quantifying social cognition and interaction in cocaine users had been rarely explored, however. Before the finalisation of the $\mathrm{ZuCo}{ }^{2} \mathrm{St}$ (Quednow, 2016), only two studies have thus far described impaired fear recognition accuracy in recreational users (Kemmis et al., 2007) and emotional intelligence deficits in dependent cocaine users (Fox et al., 2011). The $\mathrm{ZuCo}^{2} \mathrm{St}$, which started in January 2010, was the first study to examine social cognition and interaction in recreational and dependent cocaine users in depth. In contrast to the findings of the two previous studies, the $\mathrm{ZuCo}^{2} \mathrm{St}$ findings did not replicate the deficits in cocaine users in emotion perception and recognition when the stimulus material was visual (faces, complex scenes) (Preller et al., 2014b). However, the cocaine users in the $\mathrm{ZuCo}^{2} \mathrm{St}$ had problems correctly recognising the emotion from voices (prosody) and correctly linking simultaneously presented emotional faces and voices. Notably, longer duration and higher cumulative cocaine use were negatively correlated with the integration of visual and facial emotions, indicating that not only basal cognitive but also social cognitive problems of cocaine users might be at least partially drug-induced (Hulka et al., 2013). Both recreational and dependent cocaine users displayed impairments in emotional empathy, whereas only dependent users (not recreational users) revealed impaired mental perspective-taking (also called theory-of-mind), which was correlated with the severity of cocaine intake (Preller et al., 2014b).

In summary, these findings indicate disturbed emotional information integration in chronic cocaine users. Research also indicates a linkage between cocaine users' impairments in social cognition tasks and altered social reward processing (2014a). Taken together, these findings indicate that social cognition impairments in cocaine users are closely related to their real-life social functioning. Consequently, their general social competence might become impaired and promote antisocial behaviour ranging from decreased social contact to increased criminal behaviour (Preller et al., 2014a; 2014b).

\section{Neurobiological linkage between cocaine use and cognitive functioning}

Although there is increasing support for cocaine-related cognitive alterations, the exact underlying neurobiological substrates remain unclear (Cadet \& Bisagno, 2016). Adaptations involving the monoamine systems are most likely to occur as cocaine acts as an unspecific monoamine transporter blocker with high affinity for dopamine, serotonin and norepinephrine transporters (Ritz et al., 1990). Mainly owing to the higher availability of reliable radiotracers for the dopamine system, molecular imaging evidence has initially accumulated showing that addictive cocaine use leads to neuroadaptive changes in dopamine receptors and transporters in the fronto-striatal network (Hu et al., 2015; Volkow 
et al., 2004). Consequently, researchers have proposed that these changes in the dopamine system represent the common denominator of the broad cognitive impairments in cocaine users (Garavan \& Hester, 2007). However, molecular imaging studies investigating alterations in the serotonin and noradrenaline system in cocaine users are lacking, with one exception: a single positron emission tomography study has shown that norepinephrine transporter density in the thalamus is upregulated in cocaine users (Ding et al., 2010). In line with a recent publication demonstrating that increased delay discounting in cocaine users might be explained by an interaction between a genetic variation of an alpha-adrenergic receptor and cocaine use (Havranek et al., 2016), this finding suggests that adaptations in the norepinephrine system might also account for some cognitive changes found in cocaine users. Another recent study indicates that genetic variation in serotonin transporters also plays an important role in the development of working memory deficits in chronic cocaine users (Havranek et al., 2015); thus, changes in the serotonin system might be involved in cocaine-related cognitive impairments. Gaining greater insight into the various changes in the monoamine neurotransmitter systems may help explain the broad cognitive impairments of cocaine users, which are somewhat different from the more distinct cognitive deficit profiles in chronic users of other substances such as ecstasy, amphetamine and opiates (van Holst \& Schilt, 2011).

In addition, acute cocaine intake is associated with a strongly increased risk of cerebrovascular complications, such as haemorrhagic and ischaemic stroke (Treadwell \& Robinson, 2007), which often result in chronic cognitive dysfunctions (Lesniak et al., 2008). However, cognitive deficits are also observed in cocaine users without a history of strokes (Vonmoos et al., 2013).

\section{Conclusion}

The current literature suggests that the broad cognitive deficits in cocaine users might be at least partially drug-induced, and some promising results further indicate that they are potentially reversible, at least after moderate exposure and late onset of use. In addition to basal cognitive deficits, chronic cocaine users suffer from socio-cognitive and psychosocial impairments (Cadet \& Bisagno, 2016). However, there is still no clarity on the relationship between the extent of cocaine use and the development of different aspects of cognitive impairment. The decoding of this specific relationship is complex because (a) there exist some fundamental inconsistencies regarding study designs and sample characteristics (e.g. severity of use, polydrug use, psychiatric co-morbidities, main route of administration) in the current literature and (b) there are some potentially relevant modulating factors (e.g. purity of the drug, lifestyle differences, genetic predispositions, environmental factors), which are often hard to consider and have rarely been systematically investigated thus far. However, breaking down these factors is important for the personal, public and clinical management of cocaine addiction. Particularly because there is still no effective pharmacological treatment for cocaine addiction, this research provides some helpful insights and implications with regard to the development of effective prevention and intervention processes. First, basal and social cognitive functioning are closely related 
to real-life functioning and, thus, are of relevance in the daily lives of users (Garavan \& Hester, 2007; Preller et al., 2014b; Verdejo-Garcia, 2014; Volkow et al., 2011). Second, the indicated relevance of an early age-of-onset and the limited cognitive capacity of the target group suggest that the best way to tackle cocaine addiction is through prevention in an early, non-dependent stage of use and through simplified education that aims to suppress the motivational impact of drug cues. Third, in recent years, mounting evidence has suggested that cognitive impairments decrease treatment compliance and retention in cocaine-dependent patients (Aharonovich et al., 2006; Streeter et al., 2008). Additionally, Bickel et al. (2011) demonstrated that cognitive training of working memory decreases delay discounting, a fundamental feature of cocaine addiction (Hulka et al., 2014). Garcia et al. (2005) also reported that working memory and executive functions are related to more adaptive cognitive explanatory styles. Likewise, studies have shown cognitive remediation therapies to be beneficial in the treatment of other - closely related and highly co-morbid - psychiatric disorders such as schizophrenia (Green, 2005; Wykes et al., 2011), depression (Elgamal et al., 2007; Swendsen \& Merikangas, 2000) and ADHD (O'Connell et al., 2006; Wilson, 2007). Fourth, the fact that even chronic cocaine use does not necessarily lead to irreversible cognitive deficits might be a finding with an enormous impact on the motivation of affected individuals to seek treatment for their addiction. In light of the discovery of a robust cognitive profile of cocaine users and some preliminary results clearly indicating a cocaine-induced variability in cognitive performance, the next challenge is doubtlessly to clarify (a) the exact pattern and interaction of cognitive effects and their causal relationship with addiction (Garavan \& Hester, 2007) and (b) the role of other potentially relevant factors (in addition to cocaine) that might have a pivotal impact on cognitive functioning. 


\section{Mini-Dictionary of Terms}

- Cognitive functioning: The individual functional properties regarding a broad range of basal mental operations (i.e. attention, memory, executive functions).

- Emotional empathy: The ability to feel and share the emotions of others.

- Executive functions: Heterogeneous concept of complex cognitive functions usually involving the frontal cortex (inhibition, flexibility, monitoring, rule acquisition, planning etc.).

- Social cognition: Mental operations to understand oneself and others.

- Theory-of-mind: The attribution of emotions, intentions and goals to others, also known as emotional and mental perspective-taking.

\section{Key Facts of Attention-deficit/hyperactivity disorder}

- ADHD is characterised by a persistent pattern of inattention and/or hyperactivity-impulsivity.

- In DSM-V, ADHD is divided into 3 subtypes: predominantly inattentive, predominantly hyperactive/impulsive, and combined.

- ADHD symptoms may contribute to the development of substance use disorders, and, vice versa, substance use can adversely affect these symptoms (Wilson, 2007).

- The prevalence of adult ADHD in chronic cocaine users is higher than in the general population (Wunderli et al., 2016).

- Cocaine users with ADHD show stronger cognitive impairments than cocaine users without ADHD (Vonmoos et al., 2013; Wunderli et al., 2016).

\section{Summary Points}

- Recreational and dependent cocaine users display broad cognitive and socio-cognitive impairments.

- Cognitive dysfunctions in cocaine users are positively correlated with several subjective and objective drug-intake parameters and also co-vary with changing cocaine use.

- Cognitive impairments in cocaine users seem to be partially drug-induced and reversible.

- Age-of-onset of cocaine use is an important factor in cognitive impairment and recovery.

- Cognitive impairments associated with cocaine use are potentially caused by monoaminergic adaptations in the fronto-striatal network. 


\section{Figure legend}

Title Figure 1: Global cognition in controls, recreational, and dependent cocaine users.

Legend Figure 1: Global cognition scores separated for control group ( $n=68)$, recreational cocaine user group $(n=68)$, and dependent cocaine user group $(n=30)$. The dotted grey line represents the mean of the control group. The dotted black line represents the clinical criterion of -1 standard deviation of the control group (-.54; value corrected for age and verbal IQ). From Vonmoos et al. (2013) with permission from The Royal College of Psychiatrists.

Title Figure 2: Cohen's $d$ effect sizes of cognitive test scores and cognitive domains in recreational and dependent cocaine users versus controls.

Legend Figure 2: Cohen's $d$ values of recreational and dependent cocaine users compared with the control group. Data based on the z-scores (values corrected for age and verbal IQ) as reported in Vonmoos et al. (2013) with permission from The Royal College of Psychiatrists. RVP: Cantab Rapid Visual Processing, SWM: Cantab Spatial Working Memory, IED: Cantab Intra/Extradimensional Set Shifting, PAL: Cantab Paired Associates Learning, RAVLT: Rey Auditory Verbal Learning Test, LNST: Letter Number Sequencing Task. According to theoretical a priori considerations, the 15 test parameters were $z$-transformed on the basis of means/standard deviations of the control group and reduced to the four domains. Global cognition was calculated by equally integrating the four domains (for details see Vonmoos et al., 2013).

Title Figure 3: Relationship between lifetime use of cocaine and the risk for cognitive impairment.

Legend Figure 3: (a) Percentage of chronic cocaine users fulfilling the clinical cognitive criterion of below -1 standard deviation (-.54; value corrected for age and verbal IQ) in the specific cumulative dose group. (b) Percentage of chronic cocaine users fulfilling the clinical cognitive criterion of below -1 standard deviation in groups with from left to right ascending cumulative lifetime doses of cocaine. From Vonmoos et al. (2013) with permission from The Royal College of Psychiatrists.

Title Figure 4: Cognitive functions in low/high cocaine increasers and decreasers with ongoing/ceased cocaine use within one year.

Legend Figure 4: Change in global cognition score from the baseline (t1) to the one-year follow-up (t2). Follow-up values were adjusted for the test-retest effect. Mixed Ancova (corrected for ADHD) group*time interaction $\left(F_{3,33}=1.70, p=.19, p \eta^{2}=.13\right)$. Sidak post-hoc tests $\left({ }^{*} p<.05\right)$. GCI: increaser ${ }_{\text {low }} p=.61, d=-0.14$, increaser $_{\text {high }} p=.41, d=-0.30$, decreaser ${ }_{\text {ongoing }}$ $p=.82, d=0.06$, decreaser ceasing $p<.05, d=0.93$. From Vonmoos et al. (2014) with permission from Nature Publishing Group. 


\section{References}

Aharonovich, E., Hasin, D.S., Brooks, A.C., Liu, X., Bisaga, A., \& Nunes, E.V. (2006). Cognitive deficits predict low treatment retention in cocaine dependent patients. Drug and Alcohol Dependence, 81(3), 313-322.

Anthony, J.C. (2002). Epedemiology of drug dependence. In K.L. Davis, D. Charney, J.T. Coyle, \& C. Nemeroff (Eds.), Neuropsychopharmacology: The fifth generation of progress (pp. 1557-1573). Nashville: American College of Neuropsychopharmacology.

Bauer, L.O. (1996). Psychomotor and electroencephalographic sequelae of cocaine dependence. NIDA Research Monographs, 163, 66-93.

Bickel, W.K., Yi, R., Landes, R.D., Hill, P.F., \& Baxter, C. (2011). Remember the future: working memory training decreases delay discounting among stimulant addicts. Biological Psychiatry, 69(3), 260-265.

Bolla, K.I., Funderburk, F.R., \& Cadet, J.L. (2000). Differential effects of cocaine and cocaine alcohol on neurocognitive performance. Neurology, 54(12), 2285-2292.

Bolla, K.I., Rothman, R., \& Cadet, J.L. (1999). Dose-related neurobehavioral effects of chronic cocaine use. Journal of Clinical and Experimental Neuropsychology, 11(3), 361-369.

Cadet, J.L., \& Bisagno, V. (2016). Neuropsychological consequences of chronic drug use: relevance to treatment approaches. Frontiers in Psychiatry, 6.

Colzato, L.S., \& Hommel, B. (2009). Recreational use of cocaine eliminates inhibition of return. Neuropsychology, 23(1), 125-129.

Colzato, L.S., Huizinga, M., \& Hommel, B. (2009a). Recreational cocaine polydrug use impairs cognitive flexibility but not working memory. Psychopharmacology, 207(2), 225-234.

Colzato, L.S., van den Wildenberg, W.P., \& Hommel, B. (2007). Impaired inhibitory control in recreational cocaine users. PloS One, 2(11), e1143.

Colzato, L.S., van den Wildenberg, W.P., \& Hommel, B. (2009b). Reduced attentional scope in cocaine polydrug users. PloS One, 4(6), e6043.

Cooper, G.A., Kronstrand, R., \& Kintz, P. (2012). Society of Hair Testing guidelines for drug testing in hair. Forensic Science International, 218(1-3), 20-24.

Couture, S.M., Penn, D.L., \& Roberts, D.L. (2006). The functional significance of social cognition in schizophrenia: a review. Schizophrenia Bulletin, 32 Suppl 1, S44-63.

De Oliveira, L.G., Barroso, L.P., Silveira, C.M., Sanchez, Z.V., De Carvalho Ponce, J., Vaz, L.J., \& Nappo, S.A. (2009). Neuropsychological assessment of current and past crack cocaine users. Substance Use and Misuse, 44(13), 1941-1957.

Di Sclafani, V., Tolou-Shams, M., Price, L.J., \& Fein, G. (2002). Neuropsychological performance of individuals dependent on crack-cocaine, or crack-cocaine and alcohol, at 6 weeks and 6 months of abstinence. Drug and Alcohol Dependence, 66(2), 161-171.

Di Sclafani, V., Truran, D.L., Bloomer, C., Tolou-Shams, M., Clark, H.W., Norman, D., Hannauer, D., \& Fein, G. (1998). Abstinent chronic crack-cocaine and crackcocaine/alcohol abusers evidence normal hippocampal volumes on MRI despite persistent cognitive impairments. Addiction Biology, 3(3), 261-270.

Ding, Y.S., Singhal, T., Planeta-Wilson, B., Gallezot, J.D., Nabulsi, N., Labaree, D., Ropchan, J., Henry, S., Williams, W., Carson, R.E., Neumeister, A., \& Malison, R.T. (2010). PET imaging of the effects of age and cocaine on the norepinephrine transporter in the human brain using (S,S)[(11)C]O-methylreboxetine and HRRT. Synapse, 64(1), 30-38.

Elgamal, S., McKinnon, M.C., Ramakrishnan, K., Joffe, R.T., \& MacQueen, G. (2007). Successful computer-assisted cognitive remediation therapy in patients with unipolar depression: a proof of principle study. Psychological Medicine, 37(9), 1229-1238.

EMCDDA. (2015). European Monitoring Centre for Drugs and Drug Addiction. European Drug Report. Trends and Developments. 2015. Retrieved from Luxembourg:

Fox, H.C., Bergquist, K.L., Casey, J., Hong, K.A., \& Sinha, R. (2011). Selective cocaine-related difficulties in emotional intelligence: relationship to stress and impulse control. American Journal on Addictions, 20(2), 151-160.

Garavan, H., \& Hester, R. (2007). The role of cognitive control in cocaine dependence. Neuropsychology Review, 17(3), 337-345. 
García, A.V., Torrecillas, F.L., Arcos, F.A.d., \& García, M.P. (2005). Effects of executive impairments on maladaptive explanatory styles in substance abusers: clinical implications. Archives of Clinical Neuropsychology, 20(1), 67-80.

Gould, R.W., Gage, H.D., \& Nader, M.A. (2012). Effects of chronic cocaine self-administration on cognition and cerebral glucose utilization in Rhesus monkeys. Biological Psychiatry, 72(10), $856-863$

Gould, T.J. (2010). Addiction and cognition. Addiction Science \& Clinical Practice, 5(2), 4-14.

Green, A.I. (2005). Schizophrenia and comorbid substance use disorder: effects of antipsychotics. Journal of Clinical Psychiatry, 66 Suppl 6, 21-26.

Havranek, M.M., Hulka, L.M., Tasiudi, E., Eisenegger, C., Vonmoos, M., Preller, K.H., Mossner, R., Baumgartner, M.R., Seifritz, E., Grunblatt, E., \& Quednow, B.B. (2016). alpha -Adrenergic receptor polymorphisms and mRNA expression levels are associated with delay discounting in cocaine users. Addiction Biology, in press.

Havranek, M.M., Vonmoos, M., Muller, C.P., Buetiger, J.R., Tasiudi, E., Hulka, L.M., Preller, K.H., Mossner, R., Grunblatt, E., Seifritz, E., \& Quednow, B.B. (2015). Serotonin Transporter and Tryptophan Hydroxylase Gene Variations Mediate Working Memory Deficits of Cocaine Users. Neuropsychopharmacology, 40(13), 2929-2937.

Hu, Y., Salmeron, B.J., Gu, H., Stein, E.A., \& Yang, Y. (2015). Impaired functional connectivity within and between frontostriatal circuits and its association with compulsive drug use and trait impulsivity in cocaine addiction. JAMA Psychiatry, 72(6), 584-592.

Hulka, L.M., Eisenegger, C., Preller, K.H., Vonmoos, M., Jenni, D., Bendrick, K., Baumgartner, M.R., Seifritz, E., \& Quednow, B.B. (2014). Altered social and non-social decision-making in recreational and dependent cocaine users. Psychological Medicine, 44(5), 1015-1128.

Hulka, L.M., Preller, K.H., Vonmoos, M., Broicher, S.D., \& Quednow, B.B. (2013). Cocaine users manifest impaired prosodic and cross-modal emotion processing. Frontiers in Psychiatry, 4, 98.

Hyman, S.E. (2005). Addiction: a disease of learning and memory. American Journal of Psychiatry, 162(8), 1414-1422.

Jovanovski, D., Erb, S., \& Zakzanis, K.K. (2005). Neurocognitive deficits in cocaine users: a quantitative review of the evidence. Journal of Clinical and Experimental Neuropsychology, 27(2), 189-204.

Kemmis, L., Hall, J.K., Kingston, R., \& Morgan, M.J. (2007). Impaired fear recognition in regular recreational cocaine users. Psychopharmacology, 194(2), 151-159.

Koob, G.F. (2013). Addiction is a Reward Deficit and Stress Surfeit Disorder. Frontiers in Psychiatry, 4,72 .

Lesniak, M., Bak, T., Czepiel, W., Seniow, J., \& Czlonkowska, A. (2008). Frequency and prognostic value of cognitive disorders in stroke patients. Dementia and Geriatric Cognitive Disorders, 26(4), 356-363.

Magura, S., \& Kang, S.Y. (1996). Validity of self-reported drug use in high risk populations: a metaanalytical review. Substance Use and Misuse, 31(9), 1131-1153.

O'Connell, R.G., Bellgrove, M.A., Dockree, P.M., \& Robertson, I.H. (2006). Cognitive remediation in ADHD: effects of periodic non-contingent alerts on sustained attention to response. Neuropsychological Rehabilitation, 16(6), 653-665.

Porter, J.N., Olsen, A.S., Gurnsey, K., Dugan, B.P., Jedema, H.P., \& Bradberry, C.W. (2011). Chronic cocaine self-administration in rhesus monkeys: impact on associative learning, cognitive control, and working memory. Journal of Neuroscience, 31(13), 4926-4934.

Potvin, S., Stavro, K., Rizkallah, E., \& Pelletier, J. (2014). Cocaine and cognition: a systematic quantitative review. Journal of Addiction Medicine, 8(5), 368-376.

Preller, K.H., Herdener, M., Schilbach, L., Stampfli, P., Hulka, L.M., Vonmoos, M., Ingold, N., Vogeley, K., Tobler, P.N., Seifritz, E., \& Quednow, B.B. (2014a). Functional changes of the reward system underlie blunted response to social gaze in cocaine users. Proceedings of the National Academy of Sciences of the United States of America, 111(7), 2842-2847.

Preller, K.H., Hulka, L.M., Vonmoos, M., Jenni, D., Baumgartner, M.R., Seifritz, E., Dziobek, I., \& Quednow, B.B. (2014b). Impaired emotional empathy and related social network deficits in cocaine users. Addiction Biology, 19(3), 452-466. 
Quednow, B.B. (2016). The rise of the ego: social cognition and interaction in cocaine users. In V.R. Preedy (Ed.), Neuropathology of Drug Addictions and Substance Misuse (1st Edition ed., Vol. 2, pp. 257-268). London: Academic Press.

Rahman, Q., \& Clarke, C.D. (2005). Sex differences in neurocognitive functioning among abstinent recreational cocaine users. Psychopharmacology, 181(2), 374-380.

Reske, M., Eidt, C.A., Delis, D.C., \& Paulus, M.P. (2010). Nondependent stimulant users of cocaine and prescription amphetamines show verbal learning and memory deficits. Biological Psychiatry, 68(8), 762-769.

Ritz, M.C., Cone, E.J., \& Kuhar, M.J. (1990). Cocaine inhibition of ligand binding at dopamine, norepinephrine and serotonin transporters: a structure-activity study. Life Sciences, 46(9), 635645.

Rosselli, M., \& Ardila, A. (1996). Cognitive effects of cocaine and polydrug abuse. Journal of Clinical and Experimental Neuropsychology, 18(1), 122-135.

Rosselli, M., Ardila, A., Lubomski, M., Murray, S., \& King, K. (2001). Personality profile and neuropsychological test performance in chronic cocaine-abusers. International Journal of Neuroscience, 110(1-2), 55-72.

Sellaro, R., Hommel, B., \& Colzato, L.S. (2014). Increased response conflict in recreational cocaine polydrug users. Experimental Brain Research, 232(1), 113-119.

Soar, K., Dawkins, L., Page, F., \& Wooldridge, J. (2015). Recreational cocaine use is associated with attenuated latent inhibition. Addictive Behaviors, 50, 34-39.

Soar, K., Mason, C., Potton, A., \& Dawkins, L. (2012). Neuropsychological effects associated with recreational cocaine use. Psychopharmacology, 222(4), 633-643.

Spronk, D.B., van Wel, J.H., Ramaekers, J.G., \& Verkes, R.J. (2013). Characterizing the cognitive effects of cocaine: a comprehensive review. Neuroscience and Biobehavioral Reviews, 37(8), 1838-1859.

Streeter, C.C., Terhune, D.B., Whitfield, T.H., Gruber, S., Sarid-Segal, O., Silveri, M.M., Tzilos, G., Afshar, M., Rouse, E.D., Tian, H., Renshaw, P.F., Ciraulo, D.A., \& Yurgelun-Todd, D.A. (2008). Performance on the Stroop predicts treatment compliance in cocaine-dependent individuals. Neuropsychopharmacology, 33(4), 827-836.

Swendsen, J.D., \& Merikangas, K.R. (2000). The comorbidity of depression and substance use disorders. Clinical Psychology Review, 20(2), 173-189.

Treadwell, S.D., \& Robinson, T.G. (2007). Cocaine use and stroke. Postgraduate Medical Journal, 83(980), 389-394.

van Gorp, W.G., Wilkins, J.N., Hinkin, C.H., Moore, L.H., Hull, J., Horner, M.D., \& Plotkin, D. (1999). Declarative and procedural memory functioning in abstinent cocaine abusers. Archives of General Psychiatry, 56(1), 85-89.

van Holst, R.J., \& Schilt, T. (2011). Drug-related decrease in neuropsychological functions of abstinent drug users. Current Drug Abuse Reviews, 4(1), 42-56.

Verdejo-Garcia, A. (2014). Social cognition in cocaine addiction. Proceedings of the National Academy of Sciences of the United States of America, 111(7), 2406-2407.

Volkow, N.D., Baler, R.D., \& Goldstein, R.Z. (2011). Addiction: pulling at the neural threads of social behaviors. Neuron, 69(4), 599-602.

Volkow, N.D., Fowler, J.S., Wang, G.J., \& Swanson, J.M. (2004). Dopamine in drug abuse and addiction: results from imaging studies and treatment implications. Molecular Psychiatry, 9(6), 557-569.

Vonmoos, M., Hulka, L.M., Preller, K.H., Jenni, D., Baumgartner, M.R., Stohler, R., Bolla, K.I., \& Quednow, B.B. (2013). Cognitive dysfunctions in recreational and dependent cocaine users: role of attention-deficit hyperactivity disorder, craving and early age at onset. British Journal of Psychiatry, 203(1), 35-43.

Vonmoos, M., Hulka, L.M., Preller, K.H., Minder, F., Baumgartner, M.R., \& Quednow, B.B. (2014). Cognitive impairment in cocaine users is drug-induced but partially reversible: evidence from a longitudinal study. Neuropsychopharmacology, 39(9), 2200-2210.

Weightman, M.J., Air, T.M., \& Baune, B.T. (2014). A review of the role of social cognition in major depressive disorder. Frontiers in Psychiatry, 5, 179.

Wilson, J.J. (2007). ADHD and substance use disorders: developmental aspects and the impact of stimulant treatment. American Journal on Addictions, 16 Suppl 1, 5-11. 
Wunderli, M.D., Vonmoos, M., Niedecker, S.M., Hulka, L.M., Preller, K.H., Baumgartner, M.R., Kraemer, T., Seifritz, E., Schaub, M.P., Eich-Höchli, D., \& Quednow, B.B. (2016). Cognitive and emotional impairments in adults with attention-deficit/hyperactivity disorder and cocaine use. Drug and Alcohol Dependence, 1(163), 92-99.

Wykes, T., Huddy, V., Cellard, C., McGurk, S.R., \& Czobor, P. (2011). A meta-analysis of cognitive remediation for schizophrenia: methodology and effect sizes. American Journal of Psychiatry, 168(5), 472-485. 


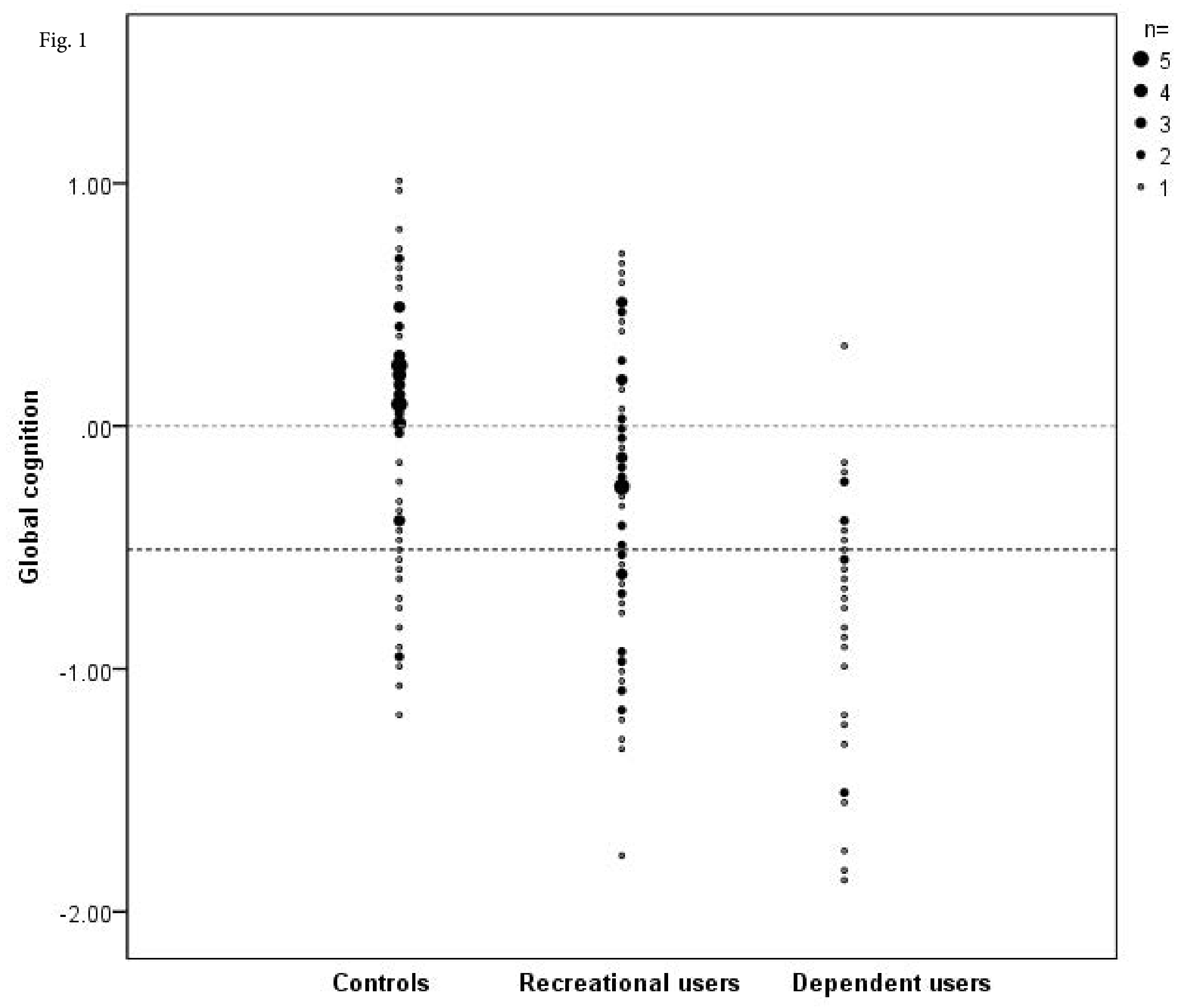


Fig. 3

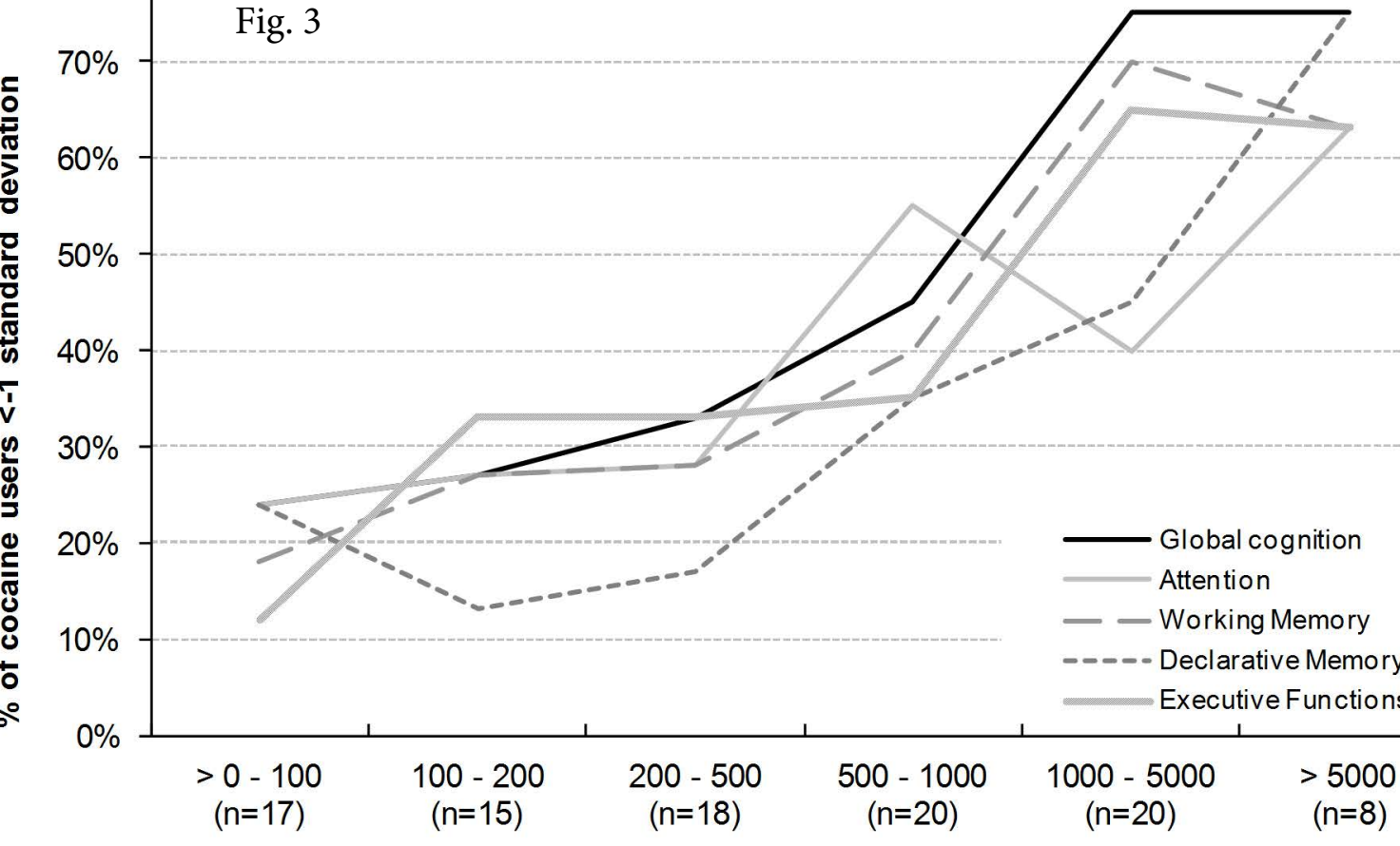

Cumulative dose (gram)

b

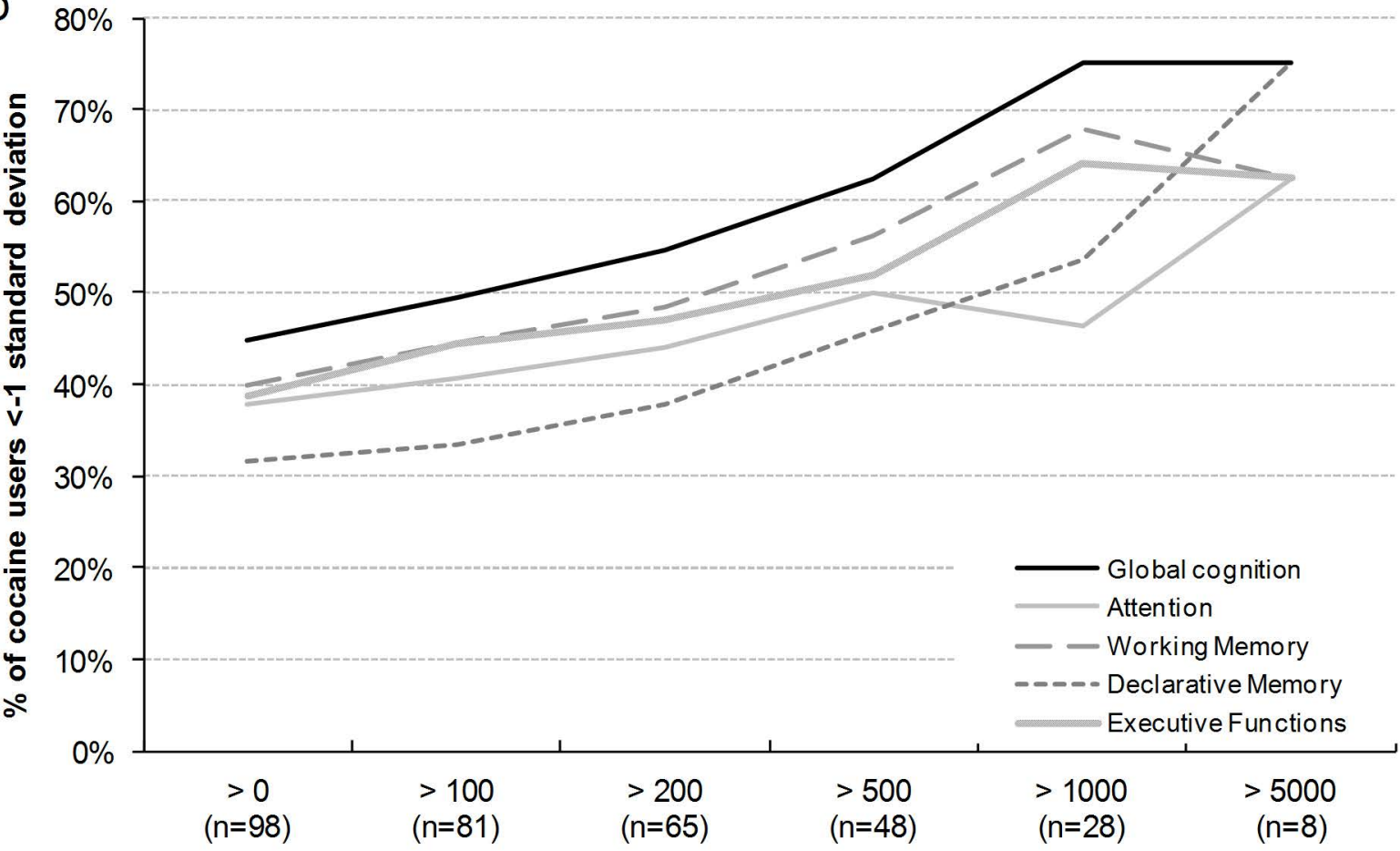

Cumulative dose (gram) 
Fig. 4

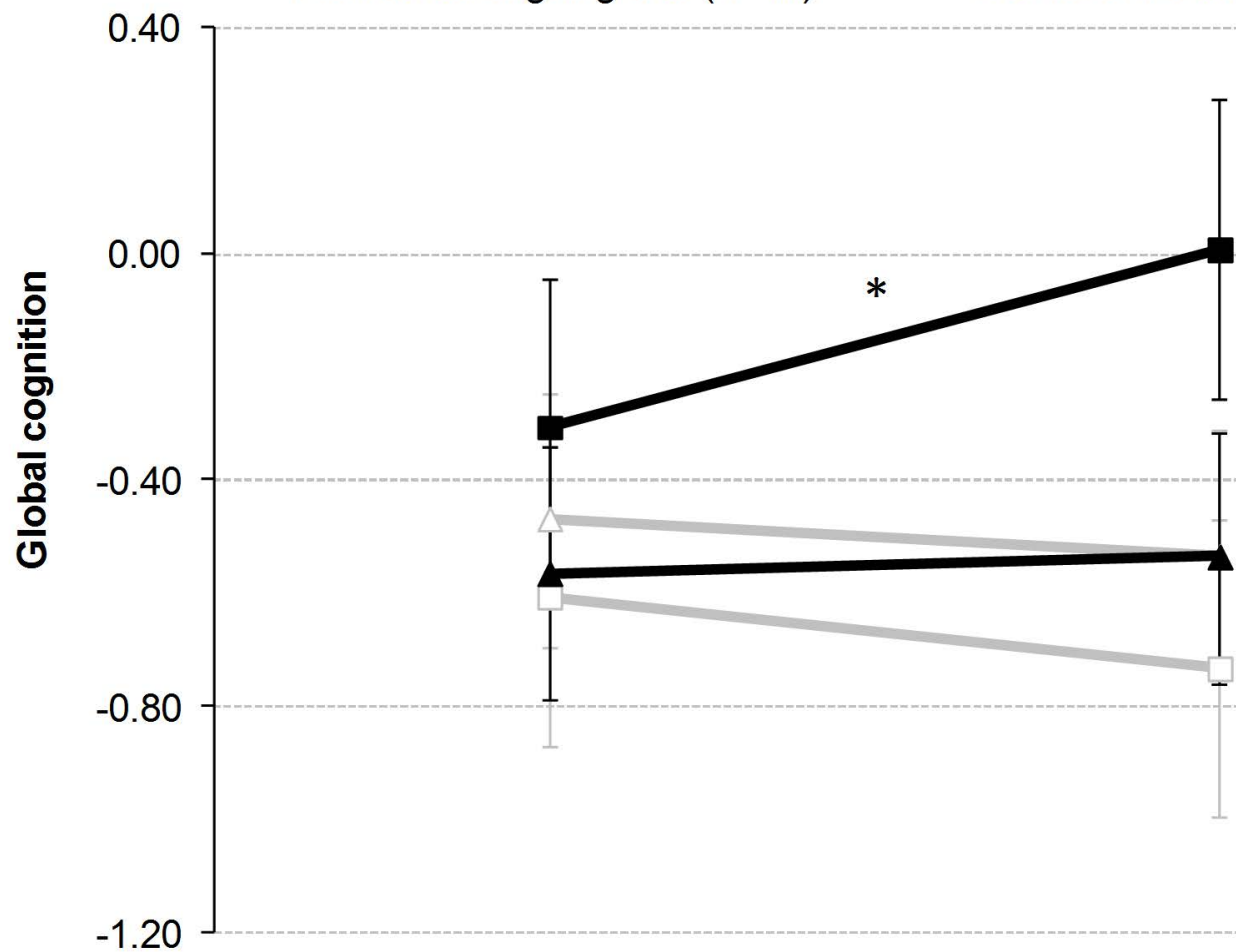

